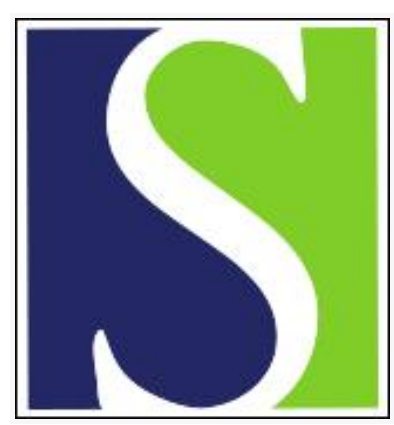

Scand J Work Environ Health 1998;24(2):81-84

https://doi.org/10.5271/sjweh.283

Issue date: Apr 1998

\title{
Bridging the generation gap
}

by Huuhtanen $P$

This article in PubMed: www.ncbi.nlm.nih.gov/pubmed/9630054

\section{(c) (1)}




\section{Bridging the generation gap}

The relationship between aging and work behavior is reciprocal. Functional capacity, values, attitudes, and motivation change as people get older and gain experience at work. Organizational and occupational status, work contents, and job demands, accordingly, change during a person's career. A person develops during his or her entire work history. In addition, the expectations toward work and occupational socialization differ among different generations and according to the phase of a person's life span.

In studies of the relationship between work and aging, the fundamental problem is how to distinguish between the effects of individual aging, age cohorts, and periodic factors. With typical crosssectional survey data on peoples' capacities and attitudes in worklife in general or in specific organizations, the impact of these factors on the results by age group is impossible to determine. The conclusions of studies on age and productivity differ if we analyze job performance in single tasks or focus on work behavior in a broader life context. Decreased capacity in single tasks can be compensated by anticipation, experience, and advanced information search strategies. In general, individual differences in capabilities and actual work performance are greater than differences between age groups.

In a constantly changing work environment, the situation is the most problematic among the lesseducated elderly employees in routine tasks. They have had fewer possibilities for career development and positive learning experiences. This situation, in turn, often leads to lower self-esteem and a lack of motivation for training. Those with less formal training and a lack of challenge during their earlier work career tend to underestimate their own capabilities and can, for this reason, have difficulties during changes at work.

Metacognition and self-concept, elderly people's own assessment of themselves as learners, can lead to a vicious circle in learning situations. Experience from both research and practice indicates that the updating of skills demands additional energy, especially from older workers. They may need more encouragement and support. Learning takes place in combining people's own long-term experience, trying out new tools, attaining experiences, and forming new concepts, mental models at work. Experienced employees have "tacit" knowledge of functional ways of organizing their daily work in local settings. Researchers have started to make a distinction between fluent and crystallized intelligence (eg, verbal capacity and reasoning, based on one's knowledge of culture). People use different learning and decision-making strategies. Among younger people these strategies are more often based on formal rules, whereas elderly workers use experience and wisdom.

For the mastery of new contents of work, the material to be learned should be fitted to one's earlier experience and expertise, and a "brush-up" of individual learning strategies may be needed. Information overload is a risk, especially under poor ergonomic conditions. With increasing age, eliminating the impacts of distractive factors in the environment, typically noise and poor lighting conditions, is more difficult. These distractions hamper concentration and the direction of attention, which in turn may lead to errors in memory function (eg, material to be learned is not processed in the short-term memory). In addition information overflow may exceed the cognitive and attention capacity of people working with visual display terminals in a multimedia environment, under conditions of continuously rising productivity demands. 
Too often people are only allowed to have a passive role in changes at work. When technological changes are in store, it is crucial that management involve employees from all age groups as active participants in the planning of the change instead of leaving the designing of information systems to technical and data experts. This interaction is one of the cornerstones of learning organizations. Users should be given the possibility to present their own proposals for the structure of the work to be computerized. Through a participatory approach, elderly people are given the possibility to control change. This ability to participate increases the feeling of self-direction and may also affect the health risks of the workers. Participation should be offered to all people in worklife, regardless of age.

There is a widespread belief that older people have a more negative attitude towards automation than younger people. Some studies have revealed differences between age groups in the ways they learn data systems. However, stereotyped attitudes dominate the discussion about aging people and computers. When new computer applications are implemented, the right timing for computer training and more time to practice under actual work conditions are crucial.

New technologies allow increased variety in tasks and operations at work. More flexible technological tools and adaptive work organizations, instead of formalized hierarchical control and tight standardization of work routines, leave room for individual variation in work capacity. This leeway provides a good opportunity for, for example, worktime arrangements and computer interfaces to be designed according to the characteristics of different user groups. Short-sighted trust in automation has led to management strategies involving a reduction of labor in the oldest age groups in companies. However, the "tacit knowledge" of elderly workers has been proved to be valuable in problem situations.

The changes in work today not only affect isolated tasks, but also more often work as a whole. The process may involve substantial implications as to one's occupational identity. The individual occupational choices and expectations of career development should be taken into account when the attitudes of workers toward changes at work are analyzed. Self-selection, training, and the recruitment of older persons to different occupations have taken place according to criteria based on worklife demands of the 1950s and 1960s. The pressure to change one's personal career orientation can be more problematic in these groups under conditions of continuous change. The adoption of an active selling role, instead of narrower clerical tasks, has been a necessity in many customer service tasks (eg, in banking and the insurance sector).

The financial squeeze of the welfare states can lead to a tougher view of the relationship between age and declining capabilities. The first step to minimize harmful competition between age groups is to disseminate unstereotyped knowledge on human aging. False assumptions and myths about the capacity of older workers must be corrected. What is needed are changes in the attitudes of both supervisors and the workers themselves. Such changes should be followed by a development of the division of tasks between age groups, a development of social support and learning, and the creation of tools for personnel administration and for occupational health and safety personnel.

Psychological life stage and life career theories emphasize the multidirectionality of personal development in adulthood, consisting of a series of transitions brought by experience. Major life events alter a person's social roles, personal identity, goals, and expectations. Decision points occur before and at the time a new role is adopted or an old one is relinquished and also at the time significant changes occur in the nature of an existing role. These changes happen both in worklife and between work and other life spheres.

The changing balance between work and retirement and the boundaries between work and nonwork have aroused much discussion and research activities. The transition from work to retirement has become less clear-cut and is now more a matter of personal timing. Better health status and the lengthening of retirement times have de-standardized the modern tripartition of the life stages. Retire- 
ment is no longer a restricted leftover period of life after "active" work. Coping with the development tasks of earlier life stages forms a basis for mastering the next stages. In this respect, work organizations have long-lasting effects on the situation of retired persons also.

In my opinion merely increasing the amount of research does not lead to a coherent picture of the complex relationship between age, work, work capacity, and the attitudes of individual persons. Too many situational, periodic, and individual factors influence this relationship, as many reviews on aging research, one in the present volume, have revealed. The well-being and productivity of elderly people at work depend both on social and labor policy at the societal and company levels. Age-conscious personnel policy means that company-specific measures should be planned for all age groups, not only for the elderly. These measures should be fitted to the specific problems, age structure, and pension policy of each organization.

To improve the situation of aging people in worklife, activities at the company level are essential. Raising the right questions is often more valuable than trying to find answers that are too simplified. It is often asked "How does aging affect learning?" It has been suggested that, instead, we should turn the question around: "How does learning affect aging?" We would then have a better starting point for improving the mental, social, and physical work demands of tomorrow. In the long run, this shift in focus will benefit workers in all age groups.

Pekka Huuhtanen

Department of Psychology

Finnish Institute of Occupational Health

Topeliuksenkatu 41 a A

FIN-00250 Helsinki Finland 